\title{
Iнформаиійні технологіi
}

\author{
УДК 658.012.011.56.005:681.3.06:665.65 \\ DOI: $10.31471 / 1993-9973-2018-2(67)-75-82$
}

\section{ДЕКОМПОЗИЦИОННЫЙ АЛГОРИТМ И МАТЕМАТИЧЕСКИЕ МОДЕЛИ ИЕРАРХИЧЕСКОЙ СТРУКТУРЫ УПРАВЛЕНИЯ ПРОЦЕССОМ ПЕРВИЧНОЙ ПЕРЕРАБОТКИ НЕФТИ}

\author{
Э.А. Меликов \\ Азербайджанский Государственный Университет Нефти и Промышленности; \\ Азадльг, 20, Баку, Насими, АZ1010
}

Розглянуто розробку детермінованих і імовірнісних моделей $і$ алгоритму управління для технологічного процесу первинної переробки нафти, а також розв'язання задачі оптимального управління в формі стохастичного програмування. Для оптимізації функиіонування досліджуваної технологічної системи методом множників Лагранжа розроблено декомпозиційний алгоритм і метод, заснований на перетворенні початкового завдання за принщипом детермінованого аналога.

Запропоновано принципи побудови оптимальної системи управління, створеної на основі розроблених моделей, алгоритму оптимізації та елементів автоматичного регулювання режимних параметрів установки первинної переробки нафти.

Ключові слова: детерміновані та імовірнісні моделі, стохастичне програмування, декомпозиційний алгоритм, метод множників Лагранжа, задача оптимізації.

Рассмотрена разработка детерминированных и вероятностных моделей и алгоритма управления для технологического процесса первичной переработки нефти, а также решение задачи оптимального управления в форме стохастического программирования. Для решения задачи оптимизации функиионирования исследуемой технологической системы методом множителей Лагранжа разработан декомпозиционный алгоритм и метод, основанный на преобразовании исходной задачи по принциипу детерминированного аналога.

Предложены приниипы построения оптимальной системы управления, созданной на основе разработанных моделей, алгоритма оптимизации и элементов автоматического регулирования режимных параметров установки первичной переработки нефти.

Ключевые слова: детерминированные и вероятностные модели, стохастическое программирование, декомпозиционный алгоритм, метод множителей Лагранжа, задача оптимизации.

The article considers the development of deterministic and probabilistic models and control algorithm for the technological process of primary oil processing, as well as the solution of the problem of optimal control in the form of stochastic programming. To solve the problem of optimization of the researched technological system functioning by means of the Lagrange multiplier method, the decomposition algorithm and a method based on the transformation of the original problem on the principle of a deterministic analog have been developed.

The principles of constructing an optimal control system based on the developed models, the optimization algorithm and the elements of automatic regulation of the regime parameters of the primary oil refining unit are proposed.

Keywords: deterministic and probabilistic models, stochastic programming, decomposition algorithm, Lagrange multiplier method, optimization problem.

Введение. В настоящее время во всем мире из-за большого спроса на топливные ресурсы важнейшими факторами повышения эффективности экономики является, прежде всего, улучшение глубины переработки нефти, улучшение качественных показателей получаемых нефтепродуктов, а также уменьшение энергетических затрат на технологических установках первичной переработки нефти. Как известно, на данных установках из сырой нефти путем подогрева в трубчатых печах до определенной температуры, а в дальнейшем ректификацией в 
колоннах получают светлые нефтяные фракции, а именно, бензин, керосин, дизельное топливо и т.д. При этом каждая из перечисленных фракций характеризуется пятью показателями качества (температурой начала кипения, температурой 5\%-го кипения, температурой 10\%-го кипения, температурой 50\%-го кипения и температурой конца кипения).

Следует отметить, что сегодня в мировой нефтеперерабатывающей и нефтехимической промышленности из-за недостаточного наличия технических средств и методов оперативного контроля вышеуказанные показатели качества светлых нефтепродуктов определяются преимущественно в заводских лабораторных условиях. Кроме того, всесторонние исследования по эксплуатации установки первичной переработки нефти типа ЭЛОУ-АВТ даже после ее модернизации показывают, что на таких технологических установках количественные и качественные показатели сырой нефти, поступающей на переработку, не являются постоянными величинами, а меняются в широком диапазоне по случайным законам [1]. Поэтому существующие локальные системы контроля и стабилизации режимных параметров, построенных по одноконтурному принципу, не могут обеспечить получение требуемых по регламенту технико-экономических показателей рассматриваемого производства.

В связи с вышеуказанными обстоятельствами, разработка аналитических моделей, осуществление математической формализации физически обоснованной задачи оптимизации с учетом стохастических особенностей координат состояния, разработка алгоритма управления и на уровне автоматического регулирования оптимальный синтез регулятора режимных координат для многоуровневой системы управления комплексом первичной переработки нефти, исходные координаты состояния и управления которого меняются по случайным законам, являются актуальными задачами.

За последние примерно 20-25 лет опубликованы весомые научные статьи, монографии, диссертации, связанные с разработкой как теоретических, так и практических сторон алгоритмического обеспечения для систем оптимального управления нефтеперерабатывающими и нефтехимическими технологическими процессами [2,3]. В частности, проблемам алгоритмизации оптимального управления и создания на их базе системы оптимального управления сложными нефтехимическими технологическими процессами, описываемыми детерминированными и нечеткими моделями посвящен ряд научных публикаций $[4,5]$.

C учетом вышеуказанных специфических особенностей функционирования первичных процессов в данной статье предлагается комплекс математических моделей и оригинальный метод оптимизации стохастических режимов на уровне оперативного управлении, а также синтез оптимального регулятора для автоматической стабилизации параметров на нижнем уровне двухуровневой системы управления ус- тановкой первичной переработки нефти типа ЭЛОУ-АВТ, функционирующего в условиях стохастической неопределенности [6].

Таким образом, тщательное исследование вопросов, связанных с разработкой систем управления сложными нефтехимическими технологическими процессами, приводит к выводу, что технологические процессы первичной переработки нефти должны относиться к классу сложных объектов, характеризующихся недостаточностью информации с позиций информационного обеспечения и по причине отсутствия контроля за показателями качества получаемых нефтяных фракций.

Постановка задачи оптимизации. В представленной статье на основе всестороннего анализа исследуемого объекта сформулирована физически обоснованная математическая постановка задачи оптимизации стохастических режимов первичных нефтеперерабатывающих технологических процессов.

Математические модели, характеризующие выход фракции и его качественные показатели, представлены в следующем виде:

$$
\begin{aligned}
& y_{k}=\bar{y}_{k}\left(x_{s}, \rho, \overline{u_{q}}\right)+\xi_{k}, \\
& v_{i}=\bar{v}_{i}\left(x_{s}, \rho, \bar{u}\right)+\xi_{i}{ }^{i} .
\end{aligned}
$$

Здесь $y_{k}$ и $v_{i}$ характеризуют соответственно расходы и качественные показатели светлых нефтяных фракций, $x_{s}$ - расход сырой нефти, поступающей на установку для переработки, $\rho$ - удельный вес сырой нефти, $\bar{u}-$ вектор управлений в рассматриваемых ректификационных колоннах (температура в различных точках, давление, уровень и т.д.), $\xi_{k}$ и $\xi_{i}^{\prime}$ величины, характеризующие погрешности, средние значения которых равны 0 .

Из выражений (1) и (2) видно, что функции

$y_{k}$ и $v_{i}$ вычисляются с определенными погрешностями. Входящие в эти выражения средние значения функций определяются посредством регрессионных зависимостей.

Как было отмечено выше, качественные показатели каждой нефтяной фракции, производимой на нефтеперерабатывающей установке, должны соответствовать определенным техническим условиям и стандартам. Эти условия математически можно представить в виде:

$$
b_{i} \leq \mathrm{v}_{i}=\overline{\mathrm{v}}_{i}\left(x_{s}, \rho, \bar{u}\right)+\xi_{i}^{\prime} \leq \overline{b_{i}}, \quad i=\overline{1, m} .
$$

Здесь $b_{i}$ и $\bar{b}_{i}-$ соответственно допустимые минимальные и максимальные значения показателей качества нефтепродуктов.

Кроме того, потенциальное количество каждой производимой на технологической установке нефтяной фракции в составе сырой нефти не должно быть меньше минимального значения, т.е.:

$$
y_{k}=\bar{y}_{k}\left(x_{s}, \rho, \bar{u}\right)+\xi_{k} \geq Q_{k}, k=\overline{1, l} .
$$


Из-за наличия случайных величин $\xi_{k}$ и $\xi_{i}{ }^{i}$ в выражениях (3) и (4), изменения параметров $x_{s}$ и $\rho$ также являются случайными величинами. Все это обуславливает вероятностный характер функциональных ограничений.

Учитывая вышесказанное, задачу оптимизации для технологического комплекса первичной переработки нефти, функционирующей в условиях неопределенности, можно представить в нижеследующем виде: при любых значениях параметров $x_{s}$ и $\rho$, характеризующих каждый из режимов рассматриваемой технологической установки, требуется в пределах установленного регламента определить такие значения управляющих параметров $\bar{u}$, при которых в этих условиях качественные показатели производимых нефтепродуктов обеспечиваются с заданной вероятностью $P_{0}$, а их количественные показатели были бы близки к потенциальной возможности содержания данной фракции в составе сырой нефти, т.е.:

$$
\begin{gathered}
P\left(\bar{y}_{k}\left(x_{s}, \rho, \bar{u}\right) \geq Q_{k}, v_{i} \in B_{i}, i=\overline{1, m},\right. \\
\left.b_{i}, \overline{b_{i}} \in B_{i} ; k=\overline{1, l}\right) \geq P_{0} .
\end{gathered}
$$

При этом значения ограничений на количество и качество целевых светлых нефтепродуктов и вероятность их выполнения задаются технологом-оператором в соответствии с регламентом данного процесса. Участие в моделях (3) и (4) случайных параметров $\xi_{k}$ и $\xi_{i}^{\prime}$, а также изменение параметров $x$ и $\rho$ по случайному закону показывает, что все вышеприведенные ограничения могут выполняться лишь с определенной степенью вероятности.

В задаче оптимизации в качестве критерия выбран максимум математического ожидания прибыли от реализации целевых нефтяных фракций:

$$
\Phi=\max _{\bar{v}_{i} \in U} E\left\{\sum_{i=1}^{N} \sum_{k \in B_{i}} c_{k}^{i} y_{k}^{i}-d(x)\right\},
$$

где $E(\cdot)$-сматематическое ожидание,

$d(x)$ - затраты на энергию, сырье и другие ресурсы на нефтеперерабатывающей установке (эта функция является линейной по отношению к переменным),

$c_{k}^{i}$ - стоимость единицы $k$-ого вида целевого светлого нефтепродукта в $i$-ом блоке.

Разработка алгоритма решения задачи оптимизации. Как видно из математической постановки (1)-(6), рассматриваемая задача оптимизации процесса первичной переработки нефти является нелинейной задачей стохастического программирования. Известно, что одним из главных и важных этапов при создании оптимальных систем управления сложными технологическими процессами является построение адекватных математических моделей составляющих установку технологических аппаратов. Опыт создания АСУ различными технологическими процессами показывает, что во многих случаях одной из основных причин их низкой эффективности является отсутствие или неполнота информации при разработке математических моделей. С этой целью при построении комплекса математических моделей основных аппаратов технологической установки типа ЭЛОУ-АВТ для сбора необходимого объема первичных технологических данных, несущих в себе более точную информацию об объекте, на рассматриваемой установке был проведен активный промышленный эксперимент. Для этого были разработаны соответствующие алгоритмы и программа. Данные, собранные в результате эксперимента были использованы при моделировании ректификационных колонн для всех видов целевых продуктов и их качественных показателей. В процессе составления моделей по отдельным координатам определены также математические ожидания, дисперсии, среднеквадратические отклонения, законы распределения и другие важные статистические характеристики. Из анализа полученных результатов установлено, что закон распределения основных координат данного процесса имеет нормальный закон распределения.

Следует отметить, что для численного решения подобной нелинейной задачи стохастического программирования в научной литературе известны приближенные и прямые методы [7-9]. Однако, это связано с весьма сложными и громоздкими вычислительными процедурами.

Установлено, что с практической точки зрения эффективным является применение метода построения детерминированного аналога. В процессе моделирования получены условные функции распределения $F\left(v_{i} / x\right)$ случайного вектора $v_{i}$. Используя их, стохастическую задачу математического программирования приведем к эквивалентной детерминированной задаче. Для этого вероятностные ограничения (5) представим в виде:

$$
F\left(v_{i} / x\right) \geq P_{0 i} .
$$

Чтобы исходную нелинейную задачу стохастического программирования свести к стандартному виду, все вероятностные ограничения заменяются на эквивалентные детерминированные:

$$
F\left(\omega_{i}\right) \geq P_{0 i} .
$$

Для численного решения рассматриваемой задачи предлагается модифицированный метод Лагранжа, учитывающий требование по выполнению условий выпуклости математических моделей. При этом, модифицированная функция Лагранжа для данной задачи оптимизации режимов атмосферной части установки первичной переработки нефти имеет следующий вид:

$$
L_{\text {mod }}(\lambda, p, x, u)=L(\lambda, x, u)+C \cdot B(p, x, u),
$$

где 


$$
\begin{gathered}
L(\lambda, x, u)=-\sum_{i=2}^{5} f_{i}\left(x^{I I}, u^{I I}, \xi^{I I}\right)-f_{1}^{I I I}\left(x^{I I I}, u^{I I I}, \xi^{I I I}\right)- \\
-\lambda_{1}\left[x_{1}^{I I}-f_{2}^{I}\left(x^{I}, u^{I}, \xi^{I}\right)\right]- \\
-\lambda_{2}\left\{\left[x_{1}^{I I I}-f_{1}^{I}\left(x^{I}, u^{I}, \xi^{I}\right)\right]-f_{1}^{I I}\left(x^{I I}, u^{I I}, \xi^{I I}\right)\right\}
\end{gathered}
$$

является классической функцией Лагранжа для данной задачи, $\lambda_{1}, \lambda_{2}-$ множители Лагранжа, $C \cdot B(p, x, u)$ - неотрицательная декомпозирующая функция. Касательно функции $B(p, x, u)$ принято нижеследующее:

$$
\begin{gathered}
B(p, x, u)=\sum_{k=I, I I, I I I} B^{j}\left(p^{k}, x^{k}, u^{k}\right), \\
B^{I}\left(p^{I}, x^{I}, u^{I}\right)=\sum_{k=1}^{2}\left(u_{k}^{I}-p_{k}^{I}\right)^{2}+\left(x^{I}-p^{I}\right)^{2}, \\
B^{I I}\left(p^{I I}, x^{I I}, u^{I I}\right)=\sum_{k=1}^{5}\left(u_{k}^{I I}-p_{k}^{I I}\right)^{2}+\left(x^{I I}-p^{I I}\right)^{2},(10 \\
B^{I I I}\left(p^{I I I}, x^{I I I}, u^{I I I}\right)=\sum_{k=1}^{2}\left(u_{k}^{I I I}-p_{k}^{I I I}\right)^{2}+\left(x^{I I I}-p^{I I I}\right)^{2},(1
\end{gathered}
$$

где

$p=\left(p^{I}, p^{I I}, p^{I I I}\right) \equiv\left(p_{1}^{I}, p_{2}^{I} ; p_{1}^{I I}, \ldots, p_{5}^{I I}, p_{1}^{I I I}, p_{2}^{I I I}\right)$ выбраны как координирующие параметры.

Функции (9)-(11) являются декомпозирующими функциями задачи (7) на локальные подзадачи меньшей размерности и обладают следующими особенностями:

1) $B(p, x, u)$ - сепарабельная функция;

2) $B\left(p^{0}, x^{0}, u^{0}\right)=0, \forall\left(x^{0}, u^{0}\right), g\left(x^{0}, u^{0}\right)=0$, где $\quad p^{0}=\left(\left(u_{1}^{I}\right)^{0},\left(u_{2}^{I}\right)^{0},\left(x^{I}\right)^{0},\left(u_{1}^{I I}\right)^{0}, \ldots,\left(u_{5}^{I I}\right)^{0}\right.$, $\left.\left(x^{I I}\right)^{0},\left(u_{1}^{I I I}\right)^{0},\left(u_{2}^{I I I}\right)^{0},\left(x^{I I I}\right)^{0}\right)$;

все миноры $\nabla^{2} B\left(p^{0}, x^{0}, u^{0}\right)$ функции Гессе являются положительными.

Поэтому, исходя из того, что $h^{\prime} \nabla^{2} B(p, x, u) h>0, \forall h$, обеспечивается строгая выпуклость функции (8).

Сущность основанного на координационном методе декомпозиционного алгоритма оптимизации состоит в том, что он включает в себя также и третий уровень, осуществляющий координирующую функцию и соединяющий в себе два других уровня. Здесь для локальных уровней формируются такие критерии, для которых, определив множители Лагранжа $\lambda$, они обеспечили бы решение задачи оптимизации и уравнения межблоковой связи на нижнем уровне.

В зависимости от результатов подзадач, решаемых на локальных уровнях, координирующий уровень формирует такие сигналы $p^{*}$, при которых решение локальных подзадач обеспечило бы решение основной задачи оптимизации функционирования установки. Учитывая вышеизложенное, для рассматриваемого состояния модифицированную функцию Лагранжа запишем в следующем виде:

$$
\begin{gathered}
L_{\text {mod }}(\lambda, x, u, p)=-\sum_{i=2}^{5} f_{i}\left(u^{I I}, x^{I I}, \xi^{I I}\right)- \\
-f_{1}^{I I I}\left(x^{I I I}, u^{I I I}, \xi^{I I I}\right)-\lambda_{1}\left(x_{1}^{I I}-f_{2}^{I}\left(x^{I}, u^{I}, \xi^{I}\right)\right)- \\
-\lambda_{2}\left(x_{1}^{I I I}-f_{1}^{I}\left(x^{I}, u^{I}, \xi^{I}\right)\right)-f_{1}^{I I I}\left(x^{I I}, u^{I I}, \xi^{I I I}\right)+ \\
+C\left(\sum_{k=1}^{2}\left(u_{k}^{I}-p_{k}^{I}\right)^{2}+\left(x^{I}-p^{I}\right)^{2}+\right. \\
+\sum_{k=1}^{5}\left(u_{k}^{I I}-p_{k}^{I I}\right)^{2}+\left(x^{I I}-p^{I I}\right)^{2}+ \\
\left.+\sum_{k=1}^{2}\left(u_{k}^{I I I}-p_{k}^{I I I}\right)^{2}+\left(x^{I I I}-p^{I I I}\right)^{2}\right) .
\end{gathered}
$$

В выражении (12), в связи с тем, что функция Лагранжа является аддитивной функцией, а декомпозирующая функция $B(p, x, u)-$ сепарабельной, при любых выбранных значениях параметров $\lambda$ и $p$ рассматриваемая задача декомпозируется на локальные подзадачи оптимизации отдельных аппаратов [10-12]. Здесь искомые переменные для аппаратов будут векторами параметров управления $\left(u^{j}\right)$ и векторами входных параметров этих аппаратов $\left(x^{j}\right)$ :

$$
\text { I. } \min _{x^{I}, u^{I} \in D} L_{\bmod _{1}}=\min _{x^{I}, u^{I}}\left[\lambda_{1} f_{2}^{I}\left(x_{0}^{I}, u^{I}, \xi^{I}\right)+\right.
$$

$\left.+\lambda_{2} f_{1}^{I}\left(x_{0}^{I}, u^{I}, \xi^{I}\right)+C\left\{\sum_{k=1}^{2}\left(u_{k}^{I}-p_{k}^{I}\right)^{2}+\left(x^{I}-p^{I}\right)^{2}\right\}\right]$

$140 \leq u_{1}^{I} \leq 160{ }^{\circ} \mathrm{C} ; 180 \leq u_{2}^{I} \leq 260{ }^{\circ} \mathrm{C} ;$

$$
660 \leq x^{I} \leq 1200 \mathrm{~m}^{3} / \text { чac. }
$$

$$
\begin{gathered}
p\left\{40=\underline{\theta}_{1}^{I} \leq x_{1 H . K}^{I} \leq \bar{\theta}_{1}^{I}=50\right\} \geq 0.94 \\
p\left\{100=\underline{\theta}_{2}^{I} \leq x_{250 \%}^{I} \leq \bar{\theta}_{2}^{I}=110\right\} \geq 0.94 \\
p\left\{170=\underline{\theta}_{3}^{I} \leq x_{3 K . K}^{I} \leq \bar{\theta}_{3}^{I}=180\right\} \geq 0.94
\end{gathered}
$$

II.

$$
\begin{aligned}
\min _{x^{I I}, u^{I I} \in D} L_{\bmod _{2}}=\min _{x^{I I}, u^{I I}}\left[\sum_{i=1}^{5} f_{i}^{I I}\left(u^{I I}, x^{I I}, \xi^{I I}\right)-\right. \\
-\lambda_{1} x^{I I}+\lambda_{2} f_{1}^{I I}\left(x^{I I}, u^{I I}, \xi^{I I}\right)+ \\
\left.+C\left\{\sum_{k=1}^{2}\left(u_{k}^{I I}-p_{k}^{I I}\right)^{2}+\left(x^{I I}-p^{I I}\right)^{2}\right\}\right]
\end{aligned}
$$

$$
105 \leq u_{1}^{I I} \leq 110{ }^{\circ} \mathrm{C} ; 330 \leq u_{2}^{I I} \leq 345{ }^{\circ} \mathrm{C} \text {; }
$$$$
120 \leq u_{3}^{I I} \leq 165{ }^{\circ} \mathrm{C} ; 160 \leq u_{4}^{I I} \leq 220{ }^{\circ} \mathrm{C} \text {; }
$$$$
230 \leq u_{5}^{I I} \leq 265{ }^{\circ} \mathrm{C} ; 800 \leq x^{I I} \leq 1200 \mathrm{~m}^{3} / \text { чac. }
$$ 


$$
\begin{gathered}
p\left\{40=\underline{\theta}_{1}^{I I} \leq x_{1 H . K}^{I I} \leq \bar{\theta}_{1}^{I I}=70\right\} \geq 0.94 \\
p\left\{100=\underline{\theta}_{2}^{I I} \leq x_{150 \%}^{I I} \leq \bar{\theta}_{2}^{I I}=115\right\} \geq 0.94 \\
p\left\{197=\underline{\theta}_{2}^{I I} \leq x_{2 H . K}^{I I} \leq \bar{\theta}_{2}^{I I}=235\right\} \geq 0.94 \\
p\left\{255=\underline{\theta}_{2}^{I I} \leq x_{250 \%}^{I I} \leq \bar{\theta}_{2}^{I I}=310\right\} \geq 0.94 \\
p\left\{305=\underline{\theta}_{2}^{I I} \leq x_{2 K . K}^{I I} \leq \bar{\theta}_{2}^{I I}=360\right\} \geq 0.94
\end{gathered}
$$

III.

$$
\begin{gathered}
\min _{x^{I I}, u^{I I I} \in D} L_{\bmod _{3}}=\min _{x^{I I}, u^{I I I}}\left[-f_{i}^{I I I}\left(u^{I I I}, x^{I I I}, \xi^{I I I}\right)-(15)\right. \\
\left.-\lambda_{2} x_{1}^{I I I}+C\left\{\sum_{k=1}^{2}\left(u_{k}^{I I I}-p_{k}^{I I I}\right)^{2}+\left(x^{I I I}-p^{I I I}\right)^{2}\right\}\right] \\
55 \leq u_{1}^{I I I} \leq 90^{\circ} C ; 130 \leq u_{2}^{I I I} \leq 205^{\circ} C ; \\
130 \leq u_{3}^{I I} \leq 305^{\circ} C \boldsymbol{M}^{3} / \text { uac. } \\
p\left\{33=\underline{\theta}_{1}^{I I I} \leq x_{1 H . K}^{I I I} \leq \bar{\theta}_{1}^{I I I}=48\right\} \geq 0.94 \\
p\left\{68=\underline{\theta}_{2}^{I I I} \leq x_{250 \%}^{I I I} \leq \bar{\theta}_{2}^{I I I}=78\right\} \geq 0.94 \\
p\left\{115=\underline{\theta}_{3}^{I I I} \leq x_{3 K . K}^{I I} \leq \bar{\theta}_{3}^{I I I}=172\right\} \geq 0.94
\end{gathered}
$$

где $\lambda_{1}, \lambda_{2}$ - неизвестные множители Лагранжа, характеризующие связи между подзадачами. В пределах указанных значений этих параметров решаются локальные подзадачи для отдельных аппаратов рассматриваемой установки:

$$
L_{j}(\lambda, p)=\min _{x^{j}, u^{j} \in D} L_{\bmod j}(\lambda, p, u, x), j=\overline{1,3} .
$$
задача:

На верхнем уровне решается следующая

$$
\max _{\lambda} L(\lambda, p)=\max _{\lambda} \min _{x^{j}, u^{j} \in D} L_{j}(\lambda, p, u, x) .
$$

Решение задачи оптимизации продолжается до тех пор, пока не выполнится нижеследующее условие:

$$
\sum_{j=1}^{3}\left[\left(u_{k}^{j}\right)^{k+1}-\left(u_{k}^{j}\right)^{k}\right]^{2}+\sum_{j=1}^{3}\left[\left(x_{k}^{j}\right)^{k+1}-\left(x_{k}^{j}\right)^{k}\right]^{2} \leq \varepsilon,
$$

где $\varepsilon$ - точность решения задачи оптимизации.

Таким образом, декомпозиционный алгоритм, обеспечивающий численное решение задачи оптимизации $(13) \div(16)$ состоит из следующих этапов:

1) с помощью градиентного метода решаются подзадачи (13) $\div(15)$;

2) проверяется выполнение условий ограничений для вероятностной характеристики температур начала, 50\%-го и конца кипения нефтепродуктов.

С этой целью:

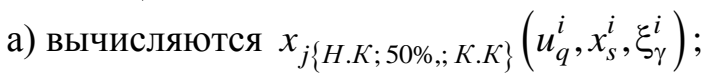

б) вычисляются $\vartheta_{j}^{i}=\frac{\theta_{j}^{i}-x_{j\{r\}}^{i}}{\sigma_{j}}$;

B) определяется $F\left(\vartheta_{j}^{i}\right)=\frac{1}{\sqrt{2 \pi}} \int_{-\infty}^{\vartheta_{j}^{i}} e^{-\frac{\vartheta_{i}^{2}}{2}} d t$

г) проверяется выполнение условия ограничений $F\left(\vartheta_{j}^{i}\right) \geq \alpha_{j}^{i}$;

3 ) определяются параметры $\lambda_{j}$, обеспечивающие выполнение материального баланса между колоннами К-1, К-2, К-8 и К-9;

4) после проверки выполнения всех условий ограничений в каждой ректификационной колонне рассчитываются оптимальные значения входных параметров и параметров управления.

В таблице 1 даны результаты решения задачи нахождения оптимальных стохастических режимов функционирования ректификационных колонн К-1 и К-2 при расходе $490 \mathrm{~m}^{3} /$ час и удельном весе 0.861 г/см сырья, поступающего на рассматриваемую установку.

На основе разработанных вероятностных моделей и декомпозиционного алгоритма оптимизации, учитывающего случайный характер входных потоков рассматриваемого объекта предложены принципы построения АСУ установкой ЭЛОУ-АВТ, представляющая собой систему с двухуровневой иерархической структурой, функционирующей в диалоговом режимe.

Функциональная структура двухуровневой системы управления атмосферным блоком рассматриваемой установки показана на рис. 1.

На верхнем уровне системы управления при любых значениях количественных и качественных показателей сырой нефти, поступающей на вход установки, определяются оптимальные рабочие режимные параметры, способные обеспечить получение нефтяных фракций в ректификационных колоннах К-1 и К-2, отвечающих стандартным требованиям показателей глубины переработки и качества нефтяных фракций. На этом уровне выбранный критерий оптимизации обеспечивается с помощью разработанных детерминированных и вероятностных математических моделей, a также декомпозиционного алгоритма оптимизации. Здесь также определяются технико-экономические показатели процесса переработки в зависимости от поступающей на установку в порядке очереди количества и качества сырой нефти.

На нижнем уровне иерархической структуры системы управления осуществляются необходимые для решения задач, предусмотренных в данной системе фильтрация режимных параметров от случайных помех, первичная переработка информации, оперативная идентификация относительно управляющих каналов, оптимальный автоматический синтез температурных параметров и непосредственное решение задачи автоматического регулирования в ко- 
Таблица 1 - Результаты решения задачи поиска оптимальных режимов функционирования колонн К-1и К-2

\begin{tabular}{|c|c|c|c|c|c|c|c|c|c|}
\hline \multirow[t]{2}{*}{$\begin{array}{l}\text { Качеств еннгіе } \\
\text { показатели } \\
\text { бензина }\end{array}$} & \multicolumn{2}{|c|}{$\begin{array}{c}\text { Расход бензина } \\
M^{3} / 4\end{array}$} & \multirow{2}{*}{$\mathcal{\sigma}_{2}{ }^{I}$} & \multirow{2}{*}{$\bar{\theta}_{2}{ }^{I}$} & \multirow{2}{*}{$\bar{y}^{I}$} & \multirow{2}{*}{$F\left(y_{2}{ }^{I} / x\right)$} & \multirow{2}{*}{$\alpha_{i}$} & \multirow{2}{*}{$u_{1}{ }^{I}$} & \multirow{2}{*}{${ }^{0}{ }^{u_{1}}{ }^{I I}$} \\
\hline & $\begin{array}{l}\text { Texy } \\
\text { щрाй }\end{array}$ & $\begin{array}{l}\text { Oпти } \\
\text { мaIвHЕIй }\end{array}$ & & & & & & & \\
\hline $\begin{array}{l}\text { Tемпература } \\
\text { начала кипения }\end{array}$ & \multirow[b]{3}{*}{38} & \multirow[b]{3}{*}{47} & 3.33 & 44 & 40.25 & 1.1 & 0.94 & \multirow[b]{3}{*}{133} & \multirow[b]{3}{*}{120} \\
\hline $\begin{array}{l}\text { Tемпература } \\
\text { 50\%-го ктпенпя }\end{array}$ & & & 5 & 116 & 107 & 1.8 & 0.94 & & \\
\hline $\begin{array}{l}\text { Tемпература } \\
\text { конша кипения }\end{array}$ & & & 10.34 & 195 & 181 & 1.4 & 0.94 & & \\
\hline
\end{tabular}

$\sigma_{2}^{I}$ - среднеквадратическое отклонение; $\bar{\theta}_{2}^{I}$ - граничное значение качественного показателя; ${\overline{y_{2}}}^{I}$ - среднее значение качественного показателя; $\alpha_{i}$ - минимальное значение вероятности; $u_{1}{ }^{I}$ и $u_{1}{ }^{I I}$ - соответственно температура верха колонн К-1 и К-2.

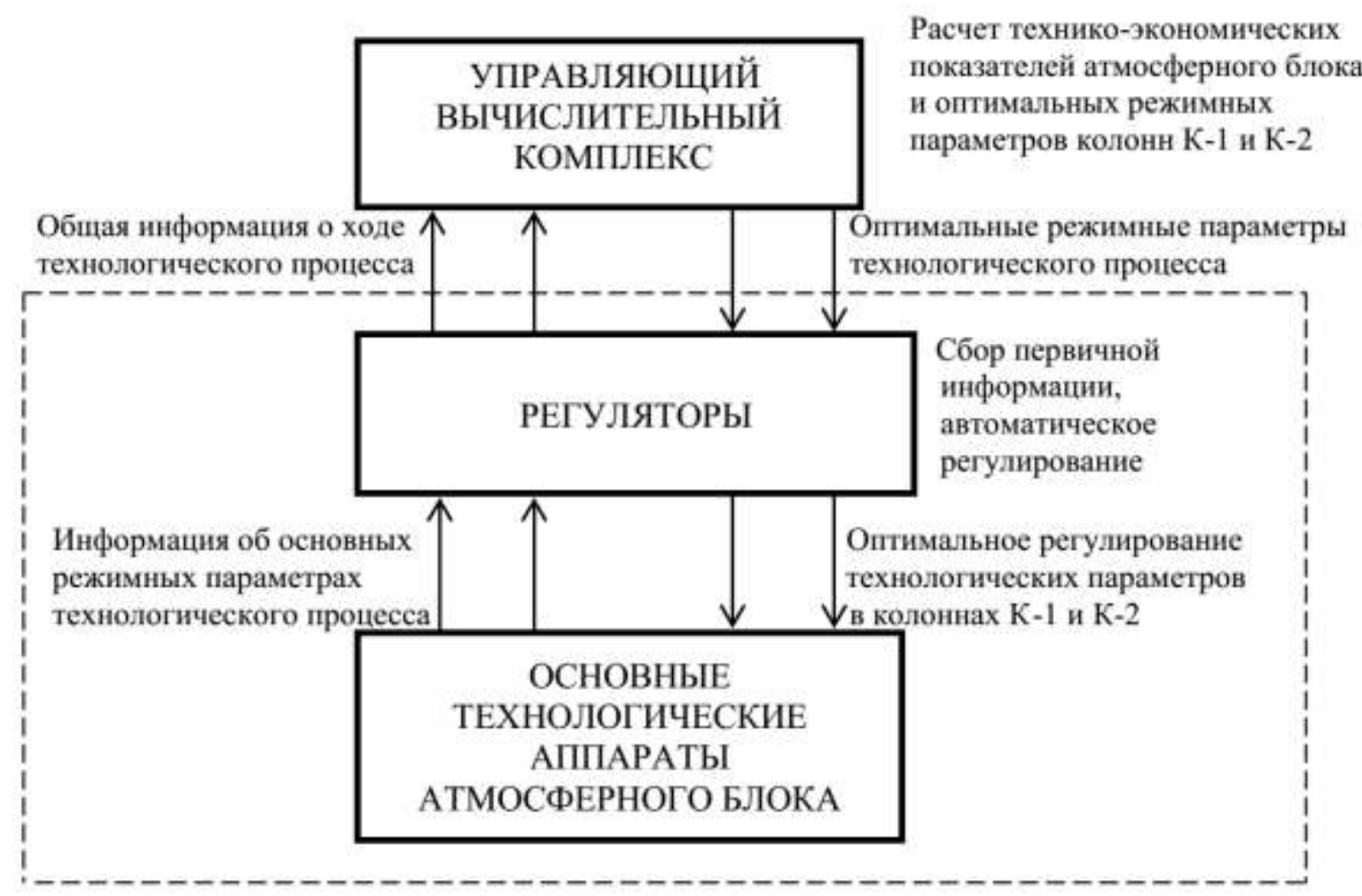

Рисунок 1 - Функциональная структура системы управления атмосферным блоком

лоннах К-1 и К-2. При этом, функционирование системы оптимального управления на каждом из его уровней осуществляется в диалоговом режиме.

В целом, функционирование рассматриваемой системы управления осуществляется как показано на рис.1: во время перехода на новый технологический режим или же изменения расхода и качественных показателей сырой нефти, поступающей на переработку на верхнем уровне, в случае необходимости обеспечения адекватности текущим ситуациям предло- женных моделей проводится адаптация с помощью определенных алгоритмов. После обеспечения адекватности моделей текущим ситуациям для определения оптимальных режимных параметров основных технологических аппаратов используется алгоритм, основанный на модифицированном методе Лагранжа. Для регулирования оптимальных значений, полученных на верхнем уровне режимных параметров, они пересылаются микропроцессорам, предусмотренных на нижнем уровне системы управления. 
Исходя из вышесказанного, на нижнем уровне оптимальной системы управления установкой первичной переработки нефти с целью стабилизации оптимальных рабочих режимных параметров технологического процесса предложено построение самонастраивающейся адаптивной системы регулирования температурных координат ректификационных колонн. Здесь с целью компенсации малых изменений, воздействующих на качественные показатели получаемых нефтяных фракций, определяется корректирующая величина, которая в зависимости от возмущений, поступающих на вход ректификационных колонн, позволяет рассчитать новое задающее воздействие, обеспечивающее оперативную реакцию даже на небольшие изменения возмущений и в дальнейшем, оперативный выход на оптимальные заданные режимы работы технологической установки с минимальными затратами.

На нижнем уровне системы управления технический персонал, используя программные и алгоритмические модули, в любой момент времени может проверить точность настроечных параметров соответствующих регуляторов температуры и также вновь оперативно вычислив оптимальные значения этих параметров и обеспечив необходимый процесс регулирования, добиваются оптимальной траектории рассматриваемого процесса управления в целом.

На нижнем уровне системы управления общая информация, связанная со сбором первичных данных о технологическом процессе, и результаты регулирования автоматически пересылаются на верхний уровень для обеспечения базы данных.

\section{Заключение}

Отличие и преимущество предлагаемой автоматической системы управления от других существующих систем $[13,14]$ заключается в обеспечении получения стабильного качества нефтяных фракций, что обеспечивается компенсацией воздействия различного рода возмущающих факторов на качественные показатели получаемых нефтепродуктов. Это осуществляется на каждом из уровней, причём на нижнем уровне малые изменения компенсируются с помощью самонастраивающихся циклов регулирования, большие же возмущения на верхнем уровне - с помощью алгоритма оптимизации стохастических режимов установки. А это, в свою очередь, по сравнению с существующими традиционными системами управления обеспечивает получение требуемого стандартного качества нефтяных фракций с минимальными энергетическими затратами.

Для оценки качественных показателей, характеризующихся температурой кипения нефтяных фракций, получаемых в колонне с использованием регулятора и условием минимизации энергетических затрат, необходимых в процессе производства, была проведена симуляция эксперимента на компьютере следующим образом: если при управлении установкой с помощью традиционной системы управления диапазон изменения температуры начала кипения бензина составляла $43-69^{0} \mathrm{C}$, температура 50\%-го кипения - $109-121{ }^{0} \mathrm{C}$, температура конца кипения $173-190{ }^{\circ} \mathrm{C}$, то в результате решения рассматриваемой задачи оптимизации с помощью синтезированного самонастраивающегося регулятора эти показатели соответственно получаются: $52-57^{0} \mathrm{C}, 110-114{ }^{0} \mathrm{C}$ и $175-180{ }^{\circ} \mathrm{C}$. Сравнительный анализ регулятора, для которого был проведен оптимальный синтез с действующей системой регулирования процесса нефтепереработки в колонне К-2, свидетельствует о том, что во втором случае, по сравнению с первым, отклонение показателей качества, характеризующегося температурой кипения нефтяных фракций, от своих оптимальных значений в среднем уменьшилось в 2-2,5 раза, что способствует снижению энергозатрат на переработку нефти на 5-7\%.

Результаты разработанного комплекса математических моделей, декомпозиционного алгоритма для решения задачи оптимизации и на их базе АСУ показали, что по сравнению с текущими режимами функционирования рассматриваемой установки предлагаемый подход и принципы автоматического регулирования режимных параметров установки обеспечивают управление комплексом первичной переработки нефти в оптимальном режиме.

\section{Литература}

1 Меликов Э.А. Задача оптимального управления технологическими процессами первичной переработки нефти / Э.А. Меликов // Материалы Международной научно-практической конференции «НЕФТЕГАЗОПЕРЕРАБОТ KA-2011». - Уфа, 2011. - C. 261-262.

2 Guseinov I.A. Nonstationary Multistage Process Control in the Petrochemical Industry / Guseinov I.A., Kurbanov Z.G., Melikov E.A., Efendiev A.I., Efendiev I.A. // Journal of Computer and Systems Sciences International, Pleiades Publishing, Ltd., 2014. - Vol. 53, No. 4. pp. 556-564.

3 Меликов Э.А. Задача оптимального распределения топливных газов между технологическими печами в нефтепереработке / Меликов Э.А., Ханбутаева Н.А. // Материалы Международной научно-практической конференции «НЕФТЕГАЗОПЕРЕРАБОТКА-2012». - Уфа, 2012. - C. 236-237.

4 Алиев Т.М. Одна задача оптимизации нестационарных реакторов / Алиев Т.М., Алиев Р.А., Эфендиев И.Р. // АН СССР. Автоматика и телемеханика. - 1975. - № 2. - С. 112 - 120 .

5 Эфендиев И.Р. Метод оптимального управления нестационарным реактором в нечетких условиях / Эфендиев И.Р., Копысицкий В.Т. // Доклады АН СССР. - 1991. - Том 318. № 3. - C. $663-667$.

6 Melikov E.A. Algorithm optimization static rejimes installation of primary oil refining / Melikov E.A., Xanbutaeva N.A. // National Science 
Review. - Chinese Academy of Sciences, Oxford University Press, 2017. - Volume 4, Issue 4 (2). pp. 1459-1466.

7 Эфендиев И.Р. Управление режимами реакторного блока производства этилена в нечетко определенных ситуациях / Эфендиев И.Р., Копысицкий В.Т. // Изв. «Российская Академия наук. Теоретические основы химической технологии». - 1993. - № 5. - С. 525 - 532.

8 Ермольев Ю.М. Методы стохастического программирования. - М.: Наука, 1976. - 340 с.

9 Аоки М. Оптимизация стохастических систем. - М.: Наука, 1971. - 424 с.

10 Родинщев Н.Е. Оптимизация управления нелинейными стохастическими системами с ограничениями / Родинщев Н.Е. // Автоматика и телемеханика, - 2001. - № 2. - С. 87 - 94.

11 Khelassi A. Analysis and Assessment of Interaction in Process Control Systems // PhD. University of Nottingham. England, 1991. 20 pp.

12 Landau I.D. System identification and control design. Englewood Cliffs. N.J.: Prentice-Hall, 1990. 20 pp.
13 Solo V., Kong X. Adaptive signal processing algorithms. Stability and Perfomance. Englewood Cliffs. N.J.: Prentice-Hall, 1995. 377 p. 14 Ибрагимов И.А. Принципы построения самообучающихся систем автоматического управления сложными технологическими процессами в условиях дефицита информации / Ибрагимов И.А., Эфендиев И.Р., Копысицкий В.Т., Меликов Э.А. // Доклады АН СССР. 1991. - Том 320. - № 6. - С. 1424 - 1427.

Стаття надійшла до редакиійної колегії 14.05.18

Рекомендована до друку професором Кондратом О.P. (ІФНТУНГ, м. Івано-Франківськ) д-ром техн. наук Ханбутаєвою Н.A. (Азербайджанський державний університет нафти і промисловості, м. Баку) 